\title{
Editorial
}

\section{Adrenergic signaling and cancer: Deciphering the connections}

\author{
Amal Melhem-Bertrandt ${ }^{\mathrm{a}, \mathrm{b}, *}$ and Anil K. Sood ${ }^{\mathrm{c}, \mathrm{d}}$ \\ ${ }^{a}$ Department of Breast medical Oncology, M.D. Anderson Cancer Center, Houston, TX, USA \\ ${ }^{\mathrm{b}}$ Breast Cancer Survivorship Clinic, M.D. Anderson Cancer Center, Houston, TX, USA \\ ${ }^{c}$ Department of Gynecologic Oncology and Cancer Biology, M.D. Anderson Cancer Center, Houston, TX, USA \\ ${ }^{\mathrm{d}}$ Center for RNA Interference and Non-Coding RNA, M.D. Anderson Cancer Center, Houston, TX, USA
}

Stress responses play a key role in adaptation to environmental, psychosocial, and physical insults. These responses are generally termed "fight or flight", because they rely on the ability of an organism to rapidly assess whether its chances of survival rely on facing or avoiding an external insult. Among the catecholamines, epinephrine (E) and norepinephrine (NE) are at the core of the "adrenergic" stress response and are quickly released following activation of the sympathetic nervous system. The other effector of the stress pathway, the hypothalamic-pituitaryadrenal axis, acts through the release of glucocorticoids (GC) [1]. When transient stress occurs, the systemic increase in these neuroendocrine hormones (E, NE, GC) promotes survival by causing appropriate physiological changes. These changes occur throughout the human body impacting cardiovascular function, growth, metabolism, reproduction, and the inflammatory/immune response $[1,2]$. However, when stress is chronic and the response maladaptive, the same stress hormones may lead to numerous pathological conditions such as cardiac, psychiatric, immunological, reproductive, and endocrine disorders [3]. Most recently,

\footnotetext{
*Corresponding author: Amal Melhem-Bertrandt, Department of Breast medical Oncology, M.D. Anderson Cancer Center, 1155 Herman Pressler Unit 1354, Houston, TX 77030, USA. Tel.: +1 713792 2817; Fax: +1 713794 4385; E-mail: amelhem@mdanderson.org.
}

the long-term effects of stress have also been shown to promote aging [4] and tumor growth and progression [5]. The details of the mechanisms by which these events occur are still unknown and are slowly unraveling.

At the cellular level, epinephrine and norepinephrine are recognized by the adrenergic receptors (ADRs) which are G-protein-coupled receptors. The main ADRs involved in the stress response are the $\alpha$ and $\beta$ ADRs. Those are membrane-bound receptors coupled to heterotrimeric guanine-binding proteins (Gproteins). The activated G-proteins dissociate from the receptor and activate intracellular targets through cAMP-dependent and independent phosphorylation events [6]. The specificity of the G-protein coupled ADRs is controlled by the type of G-protein associated with the receptor. ADRs are ubiquitously expressed throughout the body and affect numerous tissues. They have also been shown to be expressed in multiple tumor types as well as their surrounding stroma [7]. Their role in these tumor tissues has not been that of an innocent bystander, but rather an intricate involvement in driving tumor growth and progression [8]. In this issue of Cancer Biomarkers, we present contemporary epidemiologic, clinical, and preclinical data linking the adrenergic pathways and their intricate network to cancer.

In the first review, Hildegard Schuller discusses the connections between the adrenergic pathways and to- 
bacco related neoplasms with a focus on lung and pancreatic ductal adenocarcinomas. The nicotine-derived and highly carcinogenic nitrosamine (4)-methylnitrosamino-1-(3-pyridyl)-1-butanone (NNK) was found to be a high affinity agonist for $\beta$-ADRs. This, in turn, provides a putative link between activation of $\beta$ adrenergic signaling and the development of smokingassociated cancers. Furthermore, the signaling pathways activated downstream of the ADRs and their implications in pancreatic and lung cancers are discussed. This signaling cascade leads to crosstalk between the adrenergic and the epidermal growth factor receptor (EGFR) pathways, providing further mechanistic insights into the possible role this pathway has in lung cancer biology. Finally, venues for interventions are highlighted as well as potential novel biomarkers of response.

The review by Thaker et al., presents evidence linking the stress response to disruptions in reproductive physiology and to the potential development and progression of gynecologic malignancies. Their review highlights the effects of adrenergic signaling within ovarian and cervical cancer cells and within associated stroma, ultimately promoting tumor growth and metastasis. In the next chapter, Yang and Eubanks present the interplay between the neuroendocrine mediators of stress and the immune system. This has implications for the development and progression of skin malignancies, most notably melanoma. Finally, the review by Obeid and Conzen, expands on the role of adrenergic pathways in mammary tissues and presents the preclinical as well as clinical evidence linking this pathway to breast cancer. The authors also discuss how activation of the stress response can potentially induce two synergistic pathways (adrenergic and glucocorticoid), resulting in breast cancer progression. Opportunities for breast cancer prevention and treatment are highlighted with a focus on the triple negative breast cancer subtype.
Consistent and growing evidence links adrenergic pathways involved in stress response to cancer growth and progression. These mechanisms are diverse and include direct effects on DNA repair [9], cell cycle, apoptosis, migration, and invasion among others [10]. However, an indirect effect through a cross-talk with the tumor micro-environment is also apparent. Deciphering these links and elucidating some of the paradoxical results may broaden therapeutic options for patients across a multitude of tumor types.

\section{References}

[1] Chrousos GP. Stress and disorders of the stress system. Nat Rev Endocrinol. Jul 2009;5(7):374-381.

[2] Chrousos GP, Gold PW. The concepts of stress and stress system disorders. Overview of physical and behavioral homeostasis. JAMA. Mar 4 1992;267(9):1244-1252.

[3] Cohen S, Janicki-Deverts D, Miller GE. Psychological stress and disease. JAMA. Oct 10 2007;298(14):1685-1687.

[4] Lin J, Epel E, Blackburn E. Telomeres and lifestyle factors: roles in cellular aging. Mutat Res. Feb 1 2012;730(1-2):85-89.

[5] Lutgendorf SK, Sood AK, Antoni MH. Host factors and cancer progression: biobehavioral signaling pathways and interventions. J Clin Oncol. Sep 10;28(26):4094-4099.

[6] Carrasco GA, Van de Kar LD. Neuroendocrine pharmacology of stress. Eur J Pharmacol. Feb 28 2003;463(1-3):235-272.

[7] Antoni MH, Lutgendorf SK, Cole SW, et al. The influence of bio-behavioural factors on tumour biology: pathways and mechanisms. Nat Rev Cancer. Mar 2006;6(3):240-248.

[8] Armaiz-Pena GN, Cole SW, Lutgendorf SK, Sood AK. Neuroendocrine influences on cancer progression. Brain Behav Immun. Mar 2013;30 Suppl:S19-25.

[9] Hara MR, Kovacs JJ, Whalen EJ, et al. A stress response pathway regulates DNA damage through beta2-adrenoreceptors and beta-arrestin-1. Nature. Sep 15 2011;477(7364):349-353.

[10] Cole SW, Sood AK. Molecular pathways: beta-adrenergic signaling in cancer. Clin Cancer Res. Mar 1 2012;18(5):12011206. 